[J. Jap. Soc. Starch Sci., Vol. 23, No. 4, p. 187 196 (1976)]

\title{
Direction of Carbohydrate Research in the Near Future*
}

\author{
Roy L. WHISTLER** \\ **Department of Biochemistry, Purdue University, Lafayette, Indiana 47907 \\ Received November 5, 1976)
}

Having just attended this magnificent International Meeting which was so well organized by our kind and thoughtful Japanese friends and collaborators, I am sure we all have new thoughts for research and new concepts for the direction that general carbohydrate research will take in the near future.

It is presumptive to suggest what specific developments may occur in future carbohydrate research but a seer is not required to indicate areas where intensive research will occur. I wish to indicate some of these areas and to comment upon them.

Carbohydrate chemistry has grown mightily since its initial exploration in the hands of early workers. Carbohydrates were among the first compounds analyzed when carbon and hydrogen analysis became feasible, whereas, their molecular weight measurement needed to await the development of methodology such as that provided by the BECKMANN procedure. Subsequently, information developed rapidly through the work of men such as Emil Fischer and the theories of LEBEL and VAN'T HoFF. Today, there is a broad area of carbohydrate knowledge but with surprisingly large gaps where research is needed. Then, of course, there are the divergent probing frontiers that attract the great multitude of investigators who concentrate in these peripheral areas, leaving a few to work on the problems left behind or

*This is journal paper No. 6515 of the Purdue University Agricultural Experiment Station. intentionally bypassed by the frontiersmen. Some of the areas have been bypassed because appropriate instruments were not available and some because investigators simply did not know how to solve the problems posed.

\section{Basic chemistry}

The organic chemistry of carbohydrates is well established. Its principal advance in recent years has been through the application of reactions originally established by non-carbohydrate workers in the larger field of general organic investigations. As new reactions in organic chemistry evolve and new reaction mechanisms are elucidated it is natural to expect their application to carbohydrates. On the other hand, it is the novel, incisive development of a new reaction or the understanding of a new mechanism in carbohydrate chemistry that gives the field eminence and recognition in the minds of general chemists. One must remember that many such novel reactions have been first established in carbohydrate chemistry. For example, stereospecific displacement as illustrated in the Koenigs-Knorr reaction was first presented as a carbohydrate finding and was later taken into general organic chemistry.

The recent regiospecific reaction of KLEMER and Rodemeyer is a new and useful ${ }^{11}$ reaction transferred from other organic areas to carbohydrates. In the example given, methyl 2,3: 4,6 -di- $O$-benzylidene- $\alpha$-D-mannopyranoside is reacted with 2 moles of $n$-butyl lithium with 
cycloelimination to methyl 4,6-9-benzylidene$\alpha$-D-erythro-hexopyranoside-3-ulose. Some of the reaction proceeds to give an olefin but $70 \%$ proceeds through the eneol intermediate to the ketodeoxy sugar.

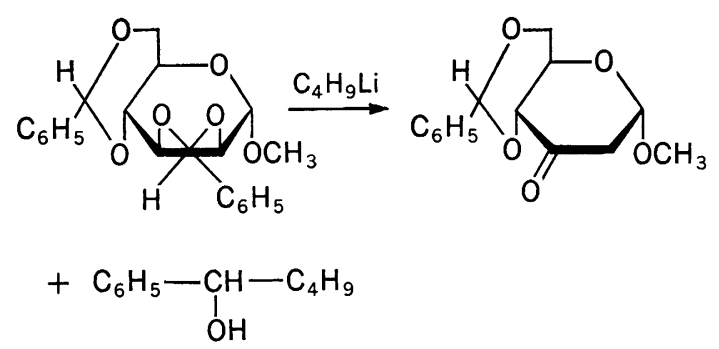

Fig. 1.

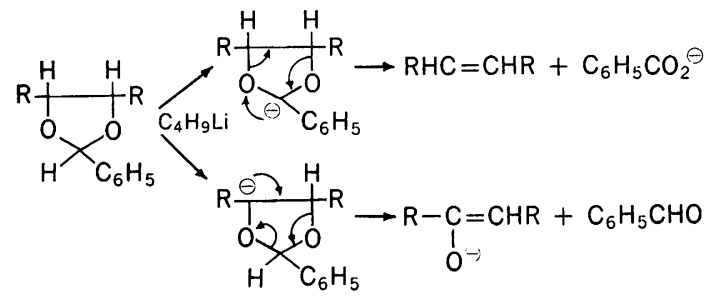

Fig. 2.

Excellent techniques for replacement of hydroxyl groups with halogen have evolved from organic chemistry. Thus, primary hydroxyl groups may be directly replaced by triphenylphosphine and N-halosuccinimides or carbon tetrachloride. ${ }^{2)}$ Another useful approach is the opening of the dioxlane ring in a 4,6-O-benzylidene hexopyranoside ${ }^{3)}$ using $\mathrm{N}$-bromosuccinimide.

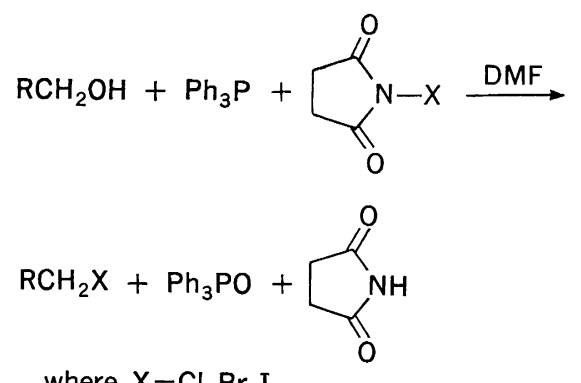

Fig. 3.

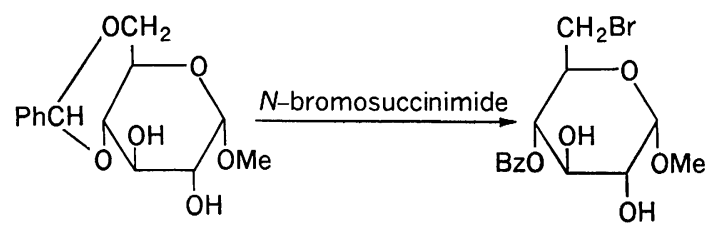

Fig. 4 .

Application of physical measurements will continue to provide data from which interpretations can be made for a better understanding of conformations and reactions. From such data more information is being obtained about the anomeric effect and the preferred orientation of the $O-1$-aglycone bond relative to the sugar ring. ${ }^{4)}$ Quantum-mechanical analysis provides also a clearer insight. ${ }^{5)}$ Cis-trans equilibra of analogus 2-mono- and 2,2-disubstituted 1,3dioxane as a function of solvent also gives valuable information. ${ }^{6)}$ The Amadori rearrangement of $\mathrm{D}$-glucose is now shown to have an equilibrium mixture of the $\beta$-pyranosyl and $\alpha, \beta$-furanosyl-amines. Lanthanide shift reagents $^{7)}$ and similar nmr shift reagents will continue to yield insights into structures and reactions. Application of mass spect:ometry and of advanced techniques in X-ray analysis are opening new approaches. Conformation of molecules in solutions and in gels is providing great insight into the understanding of molecular behavior and interaction.

\section{Antibiotic and antitumor drugs}

Carbohydrate components will continue to contribute important functions to antibiotic and antitumor drugs. This general area and especially the antitumor area may be expected to be one of the most important fields for carbohydrate research during the next 10 years.

Beginning with the first nucleotide, inosinic acid, isolated from beef extract by von LIEBIG ${ }^{8)}$ in 1847 nucleoside and nucleotide chemistry moved forward at a substantial rate until 19501953 when progress leaped upward as a result of understanding the DNA and RNA structures. This opened the door to genetic chemistry. 
KornberG ${ }^{9)}$ has said that genetic chemistry may transform the image of health and disease as dramatically as any advance in the history of medicine. In fact, there is hardly a major field of medicine that will not benefit from proper understanding of nucleosides and nucleotides. An especially heavy impact will be made against viral diseases and some diseases that are believed due to viral etiology such as arthritis, multiple sclerosis, infectious mononucleosis and leukemia. Nucleosides may effect control of parasites such as plasmodia, trypanosomes, schistosomes and ascaria. An excellent projection of future uses of nucleosides is given by RoBins as part of a recent symposium. ${ }^{10}$ SHEN ${ }^{11)}$ has pointed out interesting potential applications of nucleosides and nucleotides as therapeutic agents and BLOCK ${ }^{12}$ ) has provided insight into design and function of nucleosides.

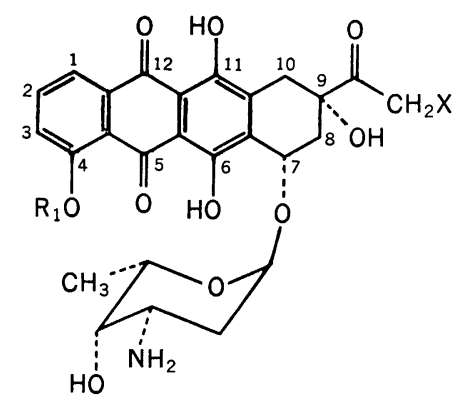

Fig. 5. Adriamycin. $\mathrm{X}=\mathrm{OH}$.

Adriamycin is perhaps the most powerful antitumor chemotherapeutic agent in use today. This drug with its near relative daunorubicin was isolated from Streptomyces peucetius var. caesius following mutagenic treatment of the parent culture with $\mathrm{N}$-nitroso-N-methylurethane. There is conscquently great activity synthetically, clinically and of a general biochemical nature to understand this drug's action and its toxicity and to improve its performance, diminish side effects and reduce its cost. The importance of its effects is attracting great interest and is bringing an avalanche of investiga- tors into every aspect of its chemistry, biochemistry and clinical medicine. Adriamycin with daunorubicin belong to the antracycline family of antibiotics. ${ }^{13-15)}$ Antitumor action is presumed due to intercalation in DNA, thereby preventing replication. A side effect is cardiomyopathy but this effect, it is hoped, can be eliminated. Total synthesis by several methods has been accomplished and the sugar moiety has been modified in various ways. One of these in which the hydroxyl at C4 has been inverted seems to be a less toxic isomer. ${ }^{15)}$

The sugar component is 3-amino-2,3,6trideoxy-L-lyxo-hexose, the same as is present in daunorubicin and similar to the 3-amino sugar in the antibiotic ristomycin ${ }^{\mathbf{1 6}}$ ) and the 3 -amino sugar in the antibiotic actinoidin. ${ }^{17-19}$ )

Macrolide antibiotics will continue to receive attention. Their synthesis appears promising and interesting since the chiral portions may be looked upon as a series of appropriately functionalized acyclic carbohydrate chains. This approach to synthesis has been given attention by Hanessian and RANCourt. ${ }^{20)}$

It is also surprising that more work has not been done on polysaccharides as antitumor agents. Very much work exists in the literature ${ }^{21)}$ to show that polysaccharides can cause tumors to regress and some polysaccharides with this property are also non-toxic. It is expected that biochemical work will be undertaken to establish how intraperatonially injected polysaccharides act upon a tumor or organize host responses to cause tumor regression.

\section{Polymers}

Since most natural carbohydrates are polysaccharides, it is reasonable to expect that a large amount of work will be done in this field. Prime objectives will be the determination of molecular conformation in solution and in gels and to determine the molecular interactions that lead to synergistic improvement in solution viscosity or gelation. A significant and 
earnest effort will be devoted to modification of polysaccharide structure to produce new molecules with improved industrial performance. Such new molecules will be obtained by new fermentation techniques wherein polysaccharides are biosynthesized or biosynthetically modified or by forming polysaccharides or modifying molecules by enzymic means. Some effort will continue to be given to chemical modification of polysaccharides.

Surely everyone will agree that the glycoprotein area will be heavily investigated during the next few years. Such work will lead to important biochemical achievements and medical benefits. Many of these benefits will derive from an understanding of the nature of cell surface recognitions, of adhesions, of viral attachment, of membranal transport and of general cell membrane and cell surface function.

Interlocking of peptidoglycans in the surfaces of both Gram-positive and Gram-negative organisms is now recognized. ${ }^{22}$ Yet to be defined, is how the cross-links are opened and
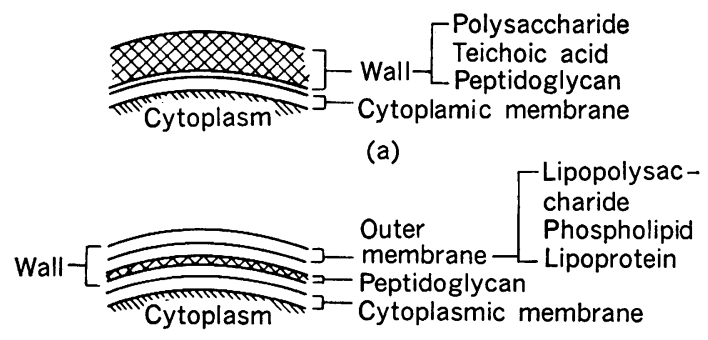

(b)

Fig. 6. Representation of the cell was of Grampositive (a) and Gram-negative (b) bacteria.

closed during cell division and in cell transformation as a result of viral invasion of the cell or as a result of oncogenesis. The actual arrangement of the carbohydrate chains, though likely perpendicular to the cell surface, requires further assessment.

Additional work is required to obtain a more complete understanding of lipopolysaccharide structure and function. Transport of the lipo- polysaccharide and its translocation to the outer membrane is in need of further effective work. Some excellent suggestions have been made by HAKOMORI and others. ${ }^{23,25)}$

In addition to purely biochemical effects the glycoproteins offer interest to the physical chemist and to the practical industrial chemist. Two examples of there interests revolve on the properties of glycoproteins in aqueous solution. The first is the unusual property of a particular glycoprotein in antarctic fish where an "antifreeze" character is provided in that the polymer inhibits the phase transition of liquid water to ice, thereby protecting the fish from death by freezing. These glycoproteins, of 11,000-

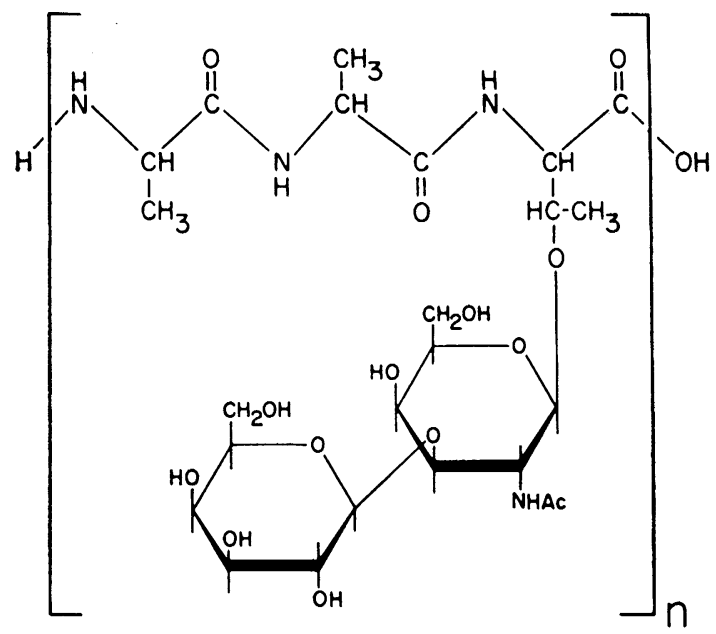

Fig. 7. Glycoprotein of artic fish.

32,000 daltons, possess the simple repeating structure of alanine-alanine-threonine, to which is attached a disaccharide unit (4-O- $\beta$-Dgalactopyranosyl-2-acetamido-2-deoxy- $\beta$-Dgalactopyranosyl). The disaccharide side chains are essential for the significant freezing point lowering of the molecule. An understanding of the specific effects of the molecule's conformation and its effect on water structures could prove of value. Understanding of a second property could provide important basic information and possibly lead to industrial uses of similar synthetic polymers. This is the property of pituitness possessed by some glycoproteins 
particularly those with numerous generally distributed oligosaccharide side chains. These molecules are able to form dispersions with long-stringy characteristics. Examples are nasal mucus or salivary discharges. Such pituitous character is desirable in certain food products and it is possible that structures with this character can be synthesized by derivatization of soy bean protein with oligosaccharides using either chemically or biochemically means to produce a covalent union.

Conformation of polysaccharides in solution is just becoming known. The excellent diffraction work on polysaccharide solutions or gels by ARNOTT and his group and the experimental work of ReEs and of many others is providing an understanding of viscosity, gel formation, synergestic actions and other rheological behavior of hyaluronic acid is more, ${ }^{26,27)}$ understandable now that it's structural conformation is known. It has taken some years to work out the structure of heparin but still more work is needed to show how the molecule functions on a molecular basis.

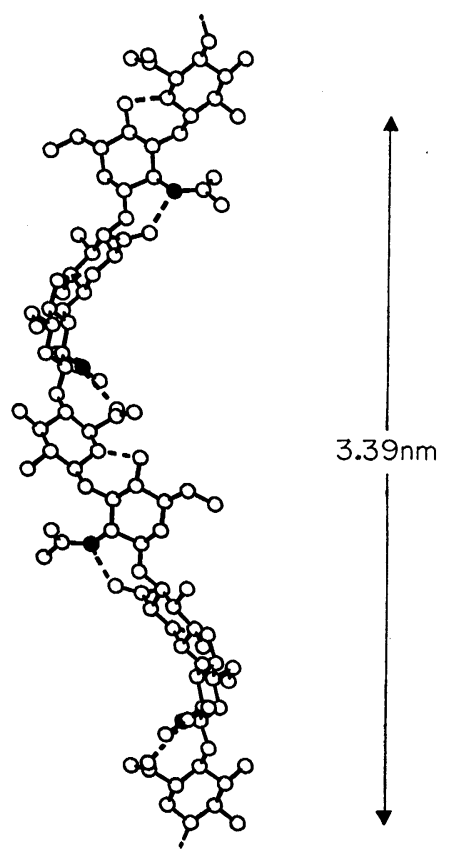

Fig. 8. Structure of hyaluronic acid (ARNOTT).

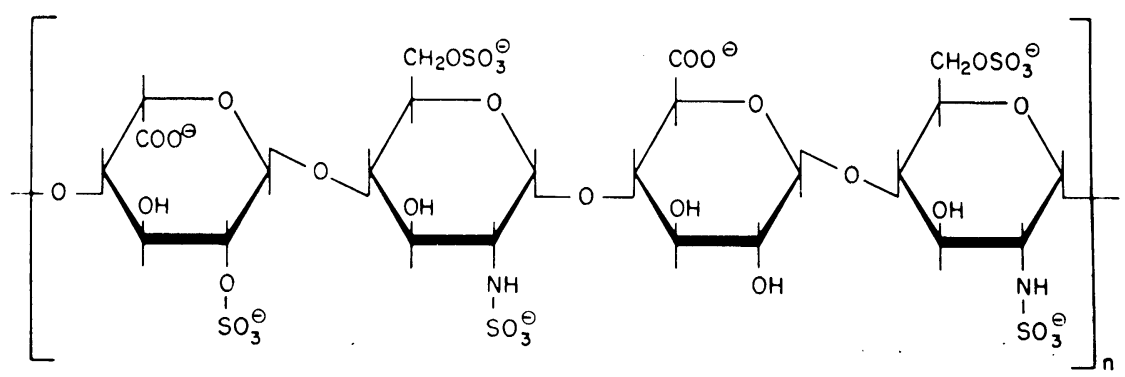

Fig. 9. Heparin.

Calcium is coordinated in an "egg crate" structure between layers of certain uronic acid containing polysaccharides. Such complex formation can explain the tight bonding of calcium and other multivalent ions in similar polysaccharide structures. It also explains how bivalent ions can cross-link such acidic polysaccharides to produce gels. The nature of calcium complexing by alginic acid is now more clearly understood.

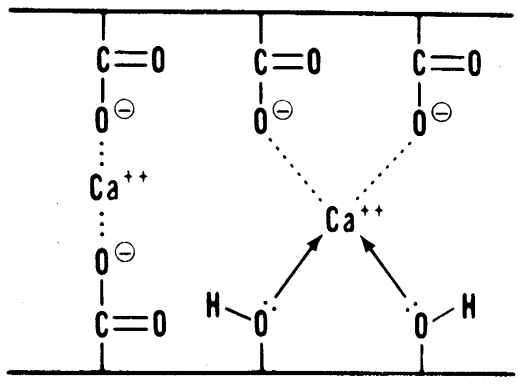

Fig. 10. Calcium complexed in polysaccharides. 


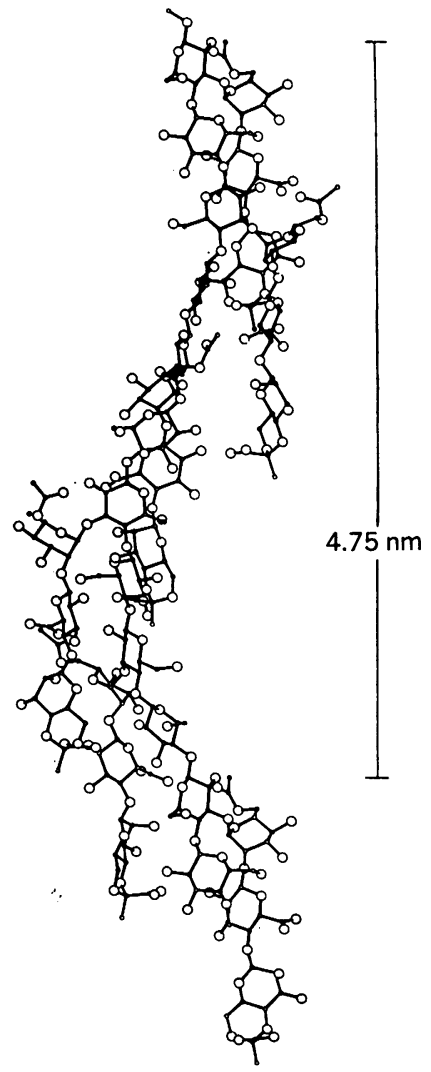

Fig. 11. Xanthan conformation (ARNotT).

Xanthan gum is known to have its side chains draped close along and spiraled around the main chain where they are held in this position by hydrogen bonds. An end view of the molecules shows it to have a furry appearance. The structure and consequently the rheological behavior of xanthan is similar to the structurally related extracellular polysaccharide of Escherichia coli. The latter differs in having 2-O(4,6-O- $\alpha$-carboxyethylidene- $\beta$-D-glucopyranosyl) $\alpha$-D-mannopyranosyl units joined $1 \rightarrow 4$ to a 4-unit main chain segment consisting of $\mathrm{D}$ mannose, D-glucose, D-glucuronic acid and Dgalactose units as shown in D-galactose/Dmannose ratio.

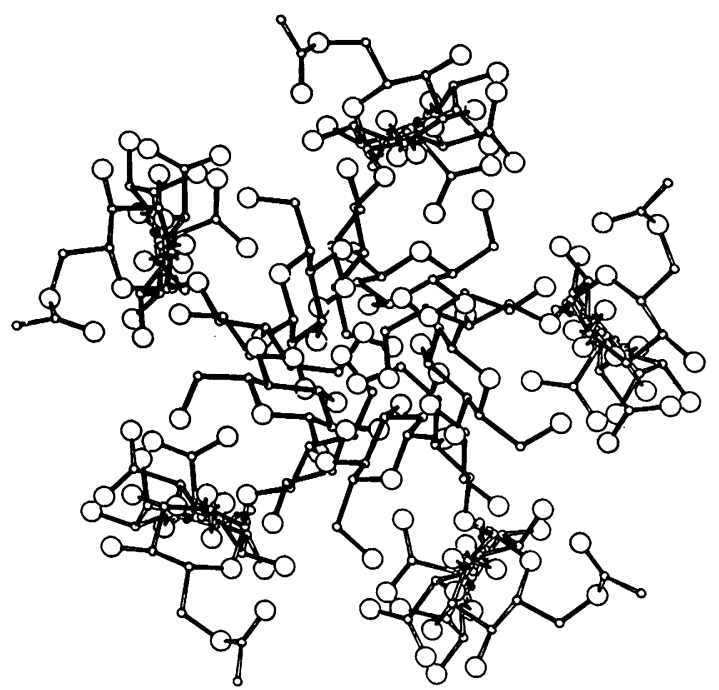

Fig. 12. Xanthan molecules as viewed from chain-end.

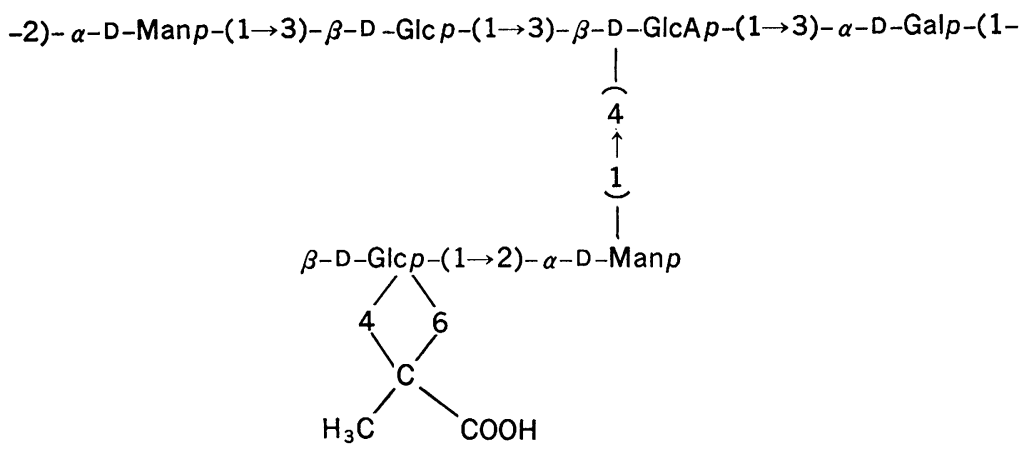

Fig. 13. Escherichia coli polysaccharide.

There is a tendency from energy considerations for polysaccharides to associate intermole- cularly. It has been known for many years that linear molecules associate in solution and, if 


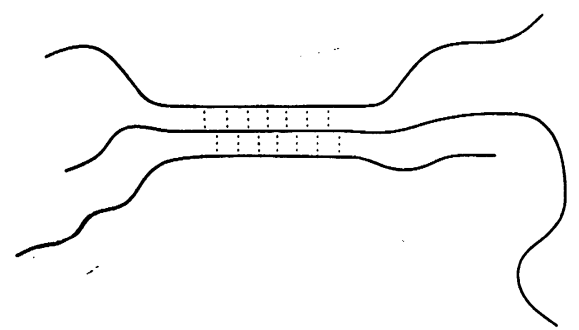

Fig. 14. Intermolecular associated of chains

neutral and the polymer is uniform in repeating units and in glycocidic bonds, the molecules will associate so high that they build groups large enough to be effected by gravitational forces and the polymer precipitates from solution. An amylose solution will so associate (retrograde) that some 99\% precipitates from solution. This retrogradation may be viewed as an attempt at crystallizations on the part of long and unwieldy molecules.

One of the principal facts to emerge from diffraction investigations is that some polysaccharides in solution tend to form a spiral of varying degrees of compactness. This is a structural form of high thermodynamic stability. In such a spiral arrangement both entropy and secondary valence forces are best satisfied.

Not only do most linear polysaccharides tend to form spirals in solution but in their tendency to associate, they may form double helices as

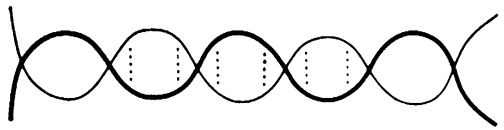

Fig. 15. Double helix.

exemplified by carrageenan. Double helices again provide structures of lower energy and high stability. Under certain conditions of concentration or temperature the double helicies may associate to form gels. Alternatively, they may associate with certain other polysaccharide molecules to form gels. This is the case of carrageenan or of xanthan in association with locust bean gum. In this synergestic relationship, locust bean gum can associate over part of its length with one double helix of carrageenan or a helix of xanthan while at the same time another portion of the locust bean gum molecule can associate with another double helix of carragreenan or a helix of xanthan. In this way molecules are cross-linked to produce a gel structure.

It was, at first, difficult to understand why locust bean gum could synergestically react with some polysaccharides to increase viscosity more than additively or to produce gels as a result of cross-linking. Both locust bean gum and guaran are galactomanns differing only in a minor way. Guaran has small or no synerges-

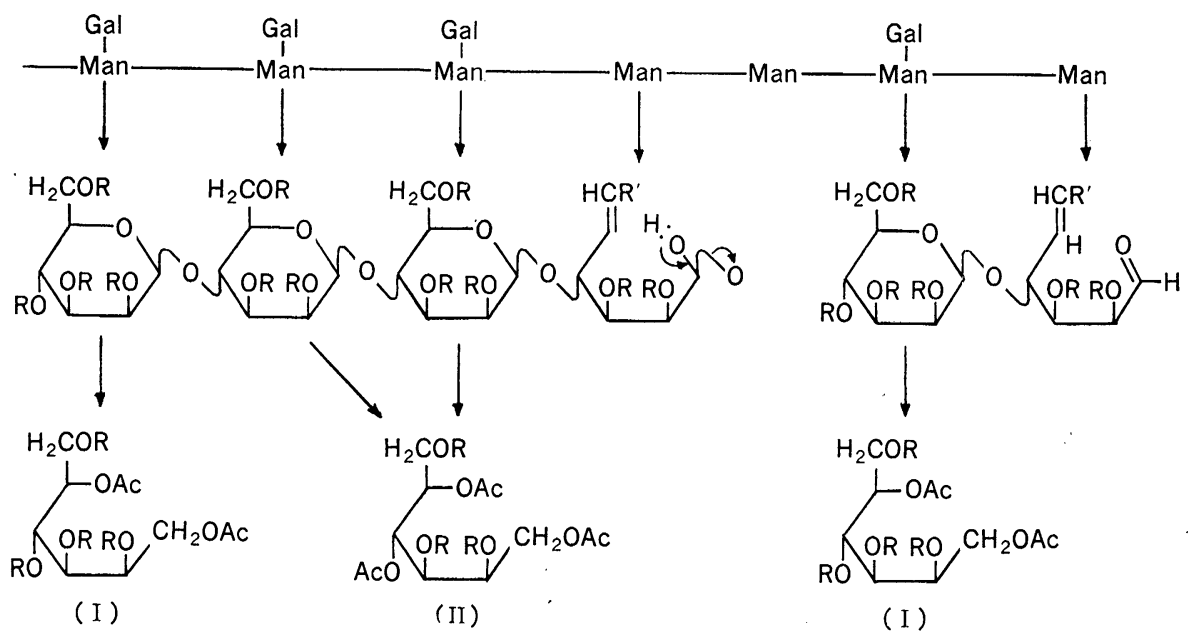

Fig. 16. Method for location of D-galactopyranosyl units on a galactomannan, 
tic effects on other polysaccharides. It was only when the distribution of the D-galactopyranosyl units was investigated that the behavioral difference between locust bean gum and guaran was brought fully to light. Thus, it was established ${ }^{28)}$ that in locust bean gum the side chains are grouped together leaving long lengths of the main chain consisting of some $85 \mathrm{D}$-mannopyranosyl units, denuded and exposed. These sections of the molecule, therefore, were free to associate with other molecules and perform the observed cross-linking effect leading to gelation. Guaran, on the other hand, has its $\alpha$-D-galactopyranosyl side chains evenly spaced on every second unit of the mannan backbone chain as was earlier established. Guaran molecules can not segmentally approach uniformly closely to each other so as to form associated regions.

Firmness or strength of gels depend on the strength of intersegmental association and the water holding capacity of the gel will depend on the spacers provided in the amorphous regions. For example, a gel of agarose is firmer than a gel of cross-linked dextran.
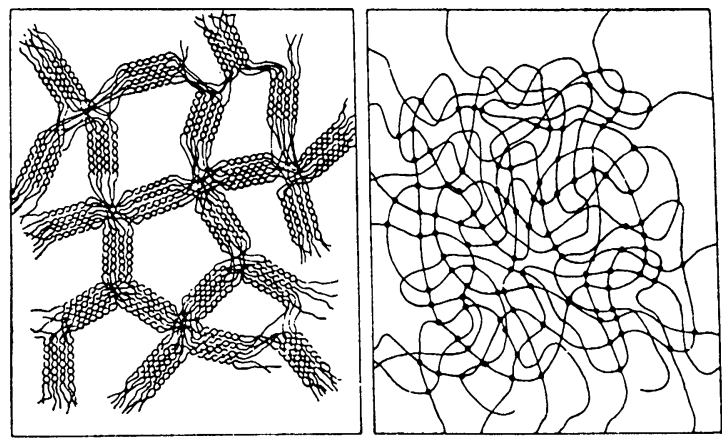

Fig. 17. Gel of agarose (Agaran) and a cross-linked dextran (Sephadex).

Curdlan is unusual in forming a "nonremelting" gel when its solution is heated. You have heard at this meeting the interesting report of Dr. T. Harada. ${ }^{29)}$ This polysaccharide has the possibility of wide usefulness. A better understanding of the molecular interactions which give rise to its rheological properties is rapidly developing and will be most useful.
More attention has been given in recent years to water absorption by solid carbohydrate structures. Not just to absorb a little water but to take up large quantities of water such as 1000-2000 times the weight of the carbohydrate. Absorbers of this mature will be of wide practical usefulness to wipe up spills, in feminine hygiene, in baby diapers, in surgical application and in common industrial applications to prevent spilling of aqueous solutions.

An interesting practical example of such an absorber is the grafted product wherein polyacrylic acid-amide is grafted to starch. ${ }^{30}$ )

Polysaccharide-protein interactions in plant cell walls

An interesting approach to an understanding of the relation between polysaccharides and proteins in plant cell walls has been made by Albersheim and coworkers. ${ }^{31-36)}$ Structural work suggests the existence of a branched arabinan and linear $(1 \rightarrow 4)$-linked galactan occurring as sidechains on a rhamnogalacturonan. Small amounts of a xyloglucan appear to be covalently linked to a number of galactan chains. Thus, the galactan appears to serve as a bridge between a xyloglucan and rhamnogalacturonan components of the wall. The rhamnogalacturonan is shown to consist of an $\alpha$-D- $(1 \rightarrow 4)$-linked galacturonan chain that is interspersed with 2-linked L-rhamnosyl residues. The L-rhamnosyl residues are not randomly distributed along the chain, but probably occur as $O$-L-rhamnosyl- $(1 \rightarrow 4)-O$-Dgalacturonosyl-( $1 \rightarrow 2)$-rhamnosyl units. This sequence appears to alternate with a homogalacturonan sequence containing approximately eight residues of 4-linked D-galacturonic acid. About half of the L-rhamnosyl residues are branched, having a substituent attached to C4, which is thought to be the likely site of the 4-linked galactan. The xyloglucan seems to be covalently linked to the pectic polysaccharide, but is non-covalently bound to the cellulose fibrils of the sycamore cell wall. The structure of this polysaccharide, on the basis of identifi- 
cation of oligosaccharides, is considered to be a repeating heptasaccharide unit consisting of four residues of $(1 \rightarrow 4)$-linked $\beta$-D-glucose and three residues of terminal $\mathrm{D}$-xylose linked singly and glycosidically to C6 of three of the D-glucosyl residues. There is evidence for a covalent connection between the pectic polysaccharides and the structural protein of the cell wall. Based on these interconnections and the strong binding which occurs between the xyloglucan and cellulose, a tentative structure of the cell wall is proposed. The model is not intended to be quantitative, but wall components are in approximately correct proportions. A very similar xyloglucan was isolated and characterized from the walls and in the extra-cellular medium of suspension-cultures of red kidneybean (Phaseolus vulgaris) cells. ${ }^{36)}$ Although some minor differences were found, the basic structure of the xyloglucans in the cell walls of these distinctly related species is the same.

It is apparent to many people that as more is learned about polysaccharide fine structure and about conformation and interacted in aqueous systems the more useful these carbohydrates will become. Consequently, industrial

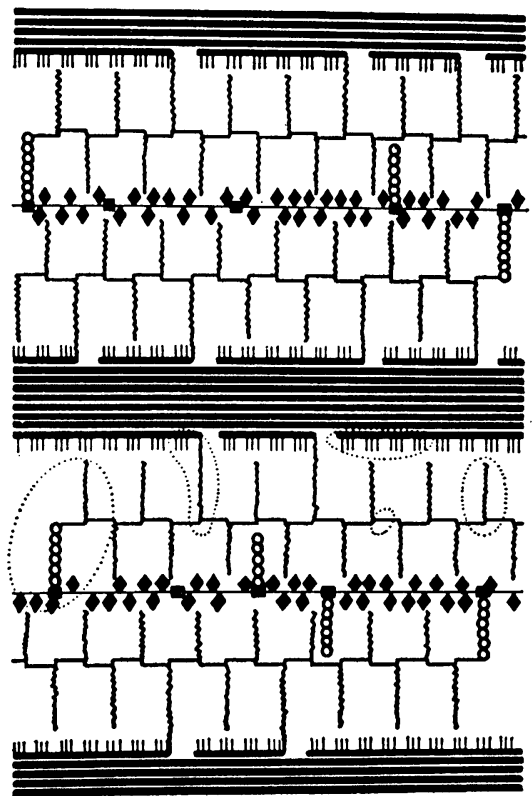

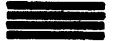

IIIIIIIIIIII

年影
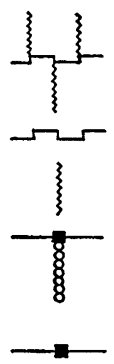

cellulose elementary fibril

xyloglucan

wall protein with arabinosyl tetrasaccharides glycosidically attached to the hydroxyproline residues

total pectic polysaccharide

rhamnogalacturonan main chain of the peptic polysaccharide

arabinan and 4-linked galactan side-chains of the peptic polymer

3,6-linked arabinogalactan attached to serine of the wall protein

unsubstituted seryl residues of the wall protein

Fig. 18. 
effort is being added to basic research work to obtain information on polysaccharides.

Coincident with this work are a growing number of projects designed to learn how to produce polysaccharide structures which will perform in expected and highly useful ways. These projects are seeking to produce new polysaccharides and to modify existing polysaccharides. The methods are chemical and biosynthetic, including microbiological and purely enzymatic. This will be an interesting and rewarding field of effort. Just to take one example, suppose a polysaccharide could be designed to work even better than xanthan in tertiary oil recovery. A polysaccharide with appropriate properties for tertiary oil recovery could be used in the United States in an estimated amount of 50 billion pounds.

\section{REFERENCES}

1) A. Klemer and G. Rodemeyer: Chem. Ber., 107, 2612 (1974).

2) S. Hanessian and P. Lavalle: Methods Carbohyd. Chem., 7, 49 (1976).

3) S. Hanessian: Methods Carbohyd. Chem., 6, 183 (1972).

4) R. Ponec and V. Chvalovsky: Coll. Czech. Chem. Commun., 39, 2613 (1974).

5) R. Ponec and V. Ghvalovsky: Coll. Czech. Chem. Commun., 39, 2613 (1974).

6) W. F. Bailey and E. L. Eliel: J. Am. Chem. Soc., 96, 1798 (1974).

7) S. B. TJan and G. A. M. van der Ouweland: Tetrahedron, 30, 2891 (1974).

8) J. von Liebig: Ann., 62, 257 (1847).

9) A. Kornberg: Science, 180, Editorial (1973).

10) Chemistry of Biology and Clinical Uses of Nucleoside Analogs, Ann. N.Y. Acad. Sci., Vol. 255, New York Acad. Sci., New York (1975).

11) T. Y. Shen: Angew. Chem., 9, 678 (1970).

12) A. Block: Drug Design, Vol. IV, Ed. E. J. Arien, Academic Press, New York (1973).

13) A. Di Marco: in Antibiotics, Vol. I, Eds. D. Gottlieb and P. P. SHAw, Springer-Verlag, Berlin, p. 190 (1966).

14) F. Arcamone, G. Franceschi, A. Minghetti, S. Penco, S. Redaello, A. DiMarco, A. M. Casazza, T. Dasdia, G. Difranzo, G. Gierliani, L. Lenaz, A. Negco and G. Sorango: J. Med. Chem., 17 335, (1975).
15) F. Arcamone, S. Penco, A. Vigevani, S. Redaelli, G. Franchi, A. Dimarco, A. M. Casazza, T. Dasdia, F. Formelli, A. Necco and C. Soranzo: J. Med. Chem., 18, 703 (1975).

16) N. N. Lomakina, L. A. Azpiridonova, R. Bognar, M. Purkas and F. Sztaricskai: Antibrotika (Moskow), 13, 975 (1968).

17) R. Bognar, F. Sztaricskai, M. E. Munk and J. Tomas: J. Org. Chem., 39, 2971 (1974).

17) F. Sztaricskai, I. Pelzras, R. Bognar and G. Bujtas: Tetrahedron Lett., 1111, (1975).

19) F. Sztaricskai, N. N. Lomakina, I. A. Spiriclonova, M. S. Frina and M. Pushkash: Antibiotics, 2, 126 (1967).

20) S. Hanessian and G. Rancourt: Cent. Am. Chem. Soc., Meeting New York, April 5-9 (1976).

21) R. L. Whistler, A. A. Bishway, P. P. Singh, W. Nakahara and R. Tokusen: Adv. Carbohydr. Chem., 32, 235 (1976).

22) L. Glaser: Ann. Rev. Biochem., 91, (1973).

23) S. Hakomori, C. G. Gahmberg, R. H. Laine and D. KIEHN: in Membrane Transformations in Neoplasma, J. Schutz and R. E. Block, Eds., Academic Press, New York, p. 297 (1974).

24) R. O. Brady and P. H. Fishman: Biochem. Biophys. Acta, 355, 121 (1974).

25) B. W. Nichols: in Plant Carbohydrate Biochemistry, Phytochem. Soc. Symp., Ser. No. 10, J. B. Pridham, Ed., Academic Press, New York, p. 97 (1974).

26) J. M. Guss, D. W. L. Kukins, P. J. C. Smith, W. T. Winters, S. Arnott, R. Moorhouse and D. A. ReEs: J. Mol. Biol., 95, 359 (1975).

27) W. T. Winter, R. J. C. Smith and S. Arnott: J. Mol. Biol., 99, 219 (1975).

28) G. W. Baker and R. L. Whistler: Carbohydr. Res., 45, 237 (1975).

29) T. Harada, M. Hisamatsu, A. Koreeda, I. Ott, T. Nakanishi, K. Kimura and S. Sato: VIII Intl. Symp. in Carbohydr. Chem., Kyoto, Japan, Aug. 16-20 (1976).

30) G. F. Fanta, F. L. Baker, R. C. Burr, W. M. DoAne and C. R. Russel: Stärke, 25, 157 (1973).

31) K. W. Talmadge, K. Keegstra, W. D. Bauer and P. Albersheim: Plant Physiol., 51, 158 (1973).

32) W. D. Bauer, K. W. Talmadge, K. Keegstra and P. Albersheim: Plant Physiol., 51, 174 (1973).

33) K. Keegstra, K. W. Talmadge, W. D. Bauer and P. Albersheim: Plant Physiol., 51, 188 (1973).

34) D. Burke, P. Kaufman, M. MaNiel and P. AlberSHEIM: Plant Physiol., 51, 109 (1974).

35) P. Albersheim: Plant Carbohydrate Biochemistry, Phytochemical Soc. Symposia, J. B. Pridham, Ed. Academic Press, New York, p. 145 (1974).

36) B. M. Wilder and P. Albersheim: Plant Physiol., 51, 889 (1973). 